\title{
Numerical Modeling of Dependent Credit Rating Transitions with Asynchronously Moving Industries
}

\author{
D. V. Boreiko' ${ }^{1}$. Y. M. Kaniovski' ${ }^{1}$ - G. Ch. Pflug ${ }^{2}$
}

\begin{abstract}
Two models of dependent credit rating migrations governed by industryspecific Markovian matrices, are considered. Caused by macroeconomic factors, positive and negative unobserved tendencies, encoded as values " 1 " or " 0 " of the corresponding variables, modify the transition probabilities and render the evolutions dependent. They are neither synchronized across industry sectors, nor over credit classes: an upswing in some of them can coexist with a decline of the rest. The models are tested on Standard and Poor's data. MATLAB optimization software and maximum likelihood estimators are used. Obtained distributions of the hidden variables demonstrate that the considered industries migrate asynchronously trough credit classes. Since downgrading probabilities are less affected by the unobserved tendencies, estimated by Monte-Carlo simulations distributions of defaults, exhibit lighter, than for the known coupling models, tails for schemes with asynchronously moving industries. Moreover, the lightest tails were obtained in the case of industry-specific transition matrices.
\end{abstract}

Keywords Macroeconomic factor - Markov process · Loss distribution · Maximum likelihood $\cdot$ Credit rating $\cdot$ Monte-Carlo simulations $\cdot$ Correlation

\footnotetext{
$凶$ Y. M. Kaniovski

YKaniovskyi@unibz.it

D. V. Boreiko

DBoreiko@unibz.it

G. Ch. Pflug

georg.pflug@univie.ac.at

1 Faculty of Economics and Management, Free University of Bozen-Bolzano, piazza Università 1, 39100 Bolzano, Italy

2 Department of Statistics and Decision Support Systems, University of Vienna, Universitätstraße 5, 1090 Vienna, Austria
} 
JEL Classification C44 · C61 · G17

\section{Introduction}

Within the CreditMetrics approach, the study of changes in the credit quality of debtors through time is a corner stone, see Gupton et al. (1997). While the credit rating of each of them evolves as a time homogeneous Markov chain, in order to model the joint distribution of a pool of debtors, a coupling scheme can be suggested. Then, introducing dependence among the migrations, the evolution of every debtor in the pool can be represented as a randomization of an idiosyncratic move and a common component. In particular, the model by Kaniovski and Pflug (2007) assumes a single common component for all debtors belonging to a credit class, while in the modification by Wozabal and Hochreiter (2012) common components are debtor-specific. Boreiko et al. (2015) study an intermediate situation when a common component remains the same for all debtors characterized by a combination of a credit class and an industry sector. In all three cases, the distribution of a common component depends on an unobserved binary tendency variable. This variable indicates whether the overall state of the economy is favorable or not for debtors belonging to the credit class in question.

In the coupling schemes quoted above, positive or negative tendencies affect uniformly all debtors having the same credit rating, regardless of their industry sectors. In other words, it is assumed that the microeconomic factors influence all industry sectors in the same way. Let us label this pattern of tendency variables as synchronous evolution of industries.

In this paper, asynchronously moving industries are analyzed. That is, tendency variables are neither synchronized across industry sectors, nor over credit classes: favorable conditions for some of them can coexist with adversities for the rest. This assumption could account better for the observed variability of the strength and of the direction of the macroeconomic factors across industry sectors. In addition, this setting allows to implement industry-specific transition matrices. This departure from the standard CreditMetrics approach may allow to model better the distributions governing credit rating migrations. For example, while estimating default correlations, default frequencies that vary across industry sectors would be a clear indication that the same Markovian matrix cannot govern migrations of all debtors. The corresponding multidimensional Markov credit rating processes and parameters defining them are specified in Sects. 2 and 3. The estimators are described in Sect. 4.

Evaluation of losses generated by a portfolio is a fundamental task of credit risk analysis. Typically the losses are quantified in terms of defaults. A range of approaches has been proposed in this case. See among others Li (2000), Nagpal and Bahar (2001), Bangia et al. (2002), Hull and White (2004), McNeil and Wendin (2007), Frydman and Schuermann (2008), Korolkiewicz and Elliott (2008), Stefanescu et al. (2009), Xing et al. (2012), Choroś-Tomczyk et al. (2013). In Sect. 7, VaR and CVaR of the distribution of the number of defaults are estimated and compared for several parameterizations of the two coupling schemes introduced here.

In order to test numerically the suggested models, a Standard and Poor's (S\&P's) data set covering OECD (Organization for Economic Co-operation and Development) 
countries is used. A pool of debtors mimicking the portfolio generating the Dow Jones iTraxx EUR index is considered. The input data are characterized in Sect. 5.

Maximum likelihood estimates were obtained by two MATLAB constrained optimization programs, the interior point algorithm (IP) and the sequential quadratic programming method (SQP). The estimates demonstrate that the considered industries evolve, in fact, asynchronously. See Sect. 6. Some implications of the asynchronicity phenomenon for credit risk assessment are presented in Sect. 7. There are MonteCarlo estimates for VaR and $\mathrm{CVaR}$ of the loss distribution and bounds for caused by macroeconomics factors variation of credit rating transition probabilities. Section 8 summarizes the obtained results. Appendix 1 contains estimated parameters. Given in Appendix 2 formulas for partial derivatives allow to accelerate substantially computations.

\section{Credit Rating Migration Process}

There is a portfolio involving debtors who are non-homogeneous in their credit ratings and who belong to different industry sectors. Let there be $M \geq 2$ non-default credit classes. Numbering them in a descending order, let us assign 1 to the most secure assets, while the next to default credit class is indexed by $M$. Defaulted debtors receive the index $M+1$. There are $S \geq 1$ industry sectors. Departing from the CreditMetrics approach (1997), where the same Markovian transition matrix applies to all debtors, let us assume that (annual) credit rating migrations in industry sector $s$ are governed by a $M \times(M+1)$ Markovian transition matrix $P^{(s)}$ with elements $p_{i, j}^{(s)}$. That is, a debtor belonging to industry sector $s$ migrates from $i$-th credit class to $j$-th with probability $p_{i, j}^{(s)}$ in one year time. Since $M+1$ is an absorption state, $p_{M+1, i}^{(s)}=\mathbb{I}_{\{i=M+1\}}$. Here $\mathbb{I}_{\{A\}}$ denotes the indicator function of a statement $A$,

$$
\mathbb{I}_{\{A\}}= \begin{cases}1 & \text { if } A \text { holds true } \\ 0 & \text { if } A \text { is false }\end{cases}
$$

The credit rating migrations occur at times $t=1,2, \ldots$ Denote by $N^{s, i}(t)$ the number of debtors from industry sector $s$ in credit class $i$ at time $t$. At the beginning there are $\mathcal{N}(1)=\sum_{i=1}^{M} \sum_{s=1}^{S} N^{s, i}(1)$ debtors in the portfolio.

A coupling technique is meant to generate a joint distribution of $N^{s, i}(1), N^{s, i}(2)$, $\ldots, N^{s, i}(t)$ such that:

- the evolutions of debtors through credit classes and industry sectors are dependent;

- the corresponding random process of credit rating transitions is time homogeneous and every individual migration in industry sector $s$ is governed by the same Markovian transition matrix $P^{(s)}$.

Assign a number $n=1,2, \ldots, \mathcal{N}(1)$ to every debtor in the portfolio at time $t=1$. Set $X_{n}(t)$ for the credit rating at time $t \geq 1$ of the debtor numbered by $n$. Then $X_{n}(t)$ is a discrete-time Markov chain with $M+1$ states. Its transient states are $1,2, \ldots, M$, while $M+1$ is an absorption state. 
The evolution of the whole portfolio is captured by a random process $\vec{X}(t)=$ $\left(X_{1}(t), X_{2}(t), \ldots, X_{\mathcal{N}(1)}(t)\right)$ whose components are stochastically dependent. Denote by $s(n)$ the industry sector of debtor $n$. The rating randomly changes in time, becoming $X_{n}(2)$ at time $t=2$, while the assignment to sector $s(n)$ remains the same. Since the distributions in question are time-homogeneous, it is enough to consider a transition from time $t=1$ to time $t=2$.

First introduce $\mathcal{N}(1)$ independent in $n$ random variables $\xi_{n}$. Each of them assumes values $1,2, \ldots, M+1$. The corresponding probabilities read:

$$
\mathbb{P}\left\{\xi_{n}=j\right\}=p_{X_{n}(1), j}^{(s(n))}
$$

Conceptually, $\xi_{n}$ represents an idiosyncratic component of the transition from $X_{n}(1)$ to $X_{n}(2)$. Its impact on the resulting move is determined by a Bernoulli random variable $\delta_{n}$ according to the formula:

$$
X_{n}(2)=\delta_{n} \xi_{n}+\left(1-\delta_{n}\right) \eta_{n}
$$

Here $\eta_{n}$ stands for a common component in the transition from $X_{n}(1)$ to $X_{n}(2)$. Taking values 1 and 0 with probabilities $q_{X_{n}(1), s(n)}$ and $1-q_{X_{n}(1), s(n)}$, random variables $\delta_{n}$ are independent in $n$. A larger value of the probability of success $q_{X_{n}(1), s(n)}$ implies less dependence of $X_{n}(2)$ on the common component. In the extreme case of $q_{X_{n}(1), s(n)} \equiv$ 1 , the credit rating of $n$-th debtor changes at $t=1$ independently of the rest of the portfolio. The families of random variables $\left\{\delta_{n}\right\},\left\{\xi_{n}\right\}$ and $\left\{\eta_{n}\right\}$ are independent.

Equation (1) is similar to what is used in common factor models. See, for example, Hull and White (2004). However, unlike them, the coefficients $\delta_{n}$ are stochastic in (1). As a consequence, the distribution of $X_{n}(2)$ is a mixture of distributions of $\xi_{n}$ and $\eta_{n}$ with weights $q_{X_{n}(1), s(n)}$ and $1-q_{X_{n}(1), s(n)}$, rather than being the convolution of them, as in the case of the common factor approach where the corresponding weights are deterministic.

Let us describe a dependence structure among common components. Denote by $\{0,1\}^{M S}$ the set of all vectors with $M S$ coordinates, each 0 or 1 . Let $\pi(\cdot)=$ $\left\{\pi(\vec{\chi}), \quad \vec{\chi} \in\{0,1\}^{M S}\right\}$ be a probability distribution. A tendency vector $\vec{\Pi}=$ $\left(\Pi_{1}, \ldots, \Pi_{M S}\right)$ is a random vector whose distribution is $\pi(\cdot)$. Coordinates $\Pi_{i}$ are not observable. They are termed as tendency, hidden or latent variables.

Let $\vec{\chi}=\left(\chi_{1}, \ldots, \chi_{M S}\right)$ be a realization of a tendency vector. Its coordinate $\chi_{M(s-1)+i}$, a realization of the tendency variable $\Pi_{M(s-1)+i}$, affects the evolution of debtors from credit class $i$ and industry sector $s$. The corresponding mechanism is described next.

When $\chi_{M(s-1)+i}=1$, all of the random variables $\eta_{n}$, such that $X_{n}(1)=i$ and $s(n)=s$, cannot assume values larger than $i$. If credit class migrations of every debtor belonging to credit class $i$ and industry sector $s$ had been governed exclusively by the corresponding $\eta_{n}$, this would have meant that the credit rating of such debtors cannot worsen. For this reason, the situation when $\chi_{M(s-1)+i}=1$ is termed as a non-deteriorating tendency for them. If $\chi_{M(s-1)+i}=0$, all of the random variables $\eta_{n}$, such that $X_{n}(1)=i$ and $s(n)=s$, take on exclusively values exceeding $i$. Had it 
been determined exclusively by the common component, the credit rating would have worsen in this case. Hence, this is a deteriorating tendency.

The conditional distribution of $\eta_{n}$ reads:

$$
\mathbb{P}\left\{\eta_{n}=j \mid \vec{\chi}\right\}=p_{X_{n}(1), j}^{(s(n))}\left(\chi_{M(s-1)+X_{n}(1)}\right),
$$

where the probabilities $p_{i, j}^{(s)}(\cdot)$ are as the following:

$$
p_{i, j}^{(s)}(1)=\left\{\begin{array}{ll}
p_{i, j}^{(s)} / p_{i}^{(s)} & \text { if } j \leq i, \\
0 & \text { if } j>i ;
\end{array} \text { and } p_{i, j}^{(s)}(0)= \begin{cases}p_{i, j}^{(s)} /\left[1-p_{i}^{(s)}\right] & \text { if } j>i, \\
0 & \text { if } j \leq i .\end{cases}\right.
$$

Here $p_{i}^{(s)}=p_{i, 1}^{(s)}+p_{i, 2}^{(s)}+\ldots+p_{i, i}^{(s)}$. This definition of conditional probabilities guarantees that each individual migration in industry sector $s$ is governed by $P^{(s)}$. It is assumed that $p_{i}^{(s)} \in(0,1)$. In fact, if $p_{i}^{(s)}=0$, then debtors from industry sector $s$ would always migrate from credit class $i$ towards more risky credit classes. Correspondingly, $p_{i}^{(s)}=1$, implies that these debtors never downgrade. (In one time instant in both cases.)

Formula (2) for conditional probabilities imply the following analytic dependence between a distribution $\pi(\cdot)$ and Markovian transition matrices $P^{(s)}$ :

$$
p_{i}^{(s)}=\sum_{\vec{\chi} \in\{0,1\}^{M S}: \chi_{M(s-1)+i}=1} \pi(\vec{\chi})
$$

These relations guarantee that the unconditional distribution of $\eta_{n}$ and, consequently the distribution of the corresponding mixture, coincides with the $X_{n}$-th row of $P^{(s(n))}$.

There are two specifications for $\left\{\eta_{n}\right\}$ termed as coupling schemes one and two in what follows next. In the first case, common components are debtor-specific. That is, conditional on $\vec{\Pi},\left\{\eta_{n}\right\}$ are independent in $n$. In the second case, the same common component applies to all debtors characterized by a combination of an industry sector and a credit class. More precisely, given $\vec{\Pi}$, random variables $\eta_{n}$ and $\eta_{l}$ are stochastically independent if $s(n) \neq s(l)$ or $X_{n}(1) \neq X_{l}(1)$, while $\eta_{n}=\eta_{l}$ if $s(n)=s(l)$ and $X_{n}(1)=X_{l}(1)$.

Counts $N^{s, i}(2)$ at time $t=2$ are obtained by the following formula:

$$
N^{s, i}(2)=\sum_{n=1}^{\mathcal{N}(1)} \mathbb{1}_{\left\{X_{n}(2)=i, s(n)=s\right\}}
$$

The total number of defaults $D(1)$ at time $t=2$ equals: 


$$
\mathcal{N}(1)-\mathcal{N}(2)=\sum_{n=1}^{\mathcal{N}(1)} \mathbb{1}_{\left\{X_{n}(2)=M+1\right\}}
$$

while the corresponding number of defaults $D^{(s)}(1)$ in industry sector $s$ reads:

$$
D^{(s)}(1)=\sum_{n=1}^{\mathcal{N}(1)} \mathbb{1}_{\{s(n)=s\}} \mathbb{1}_{\left\{X_{n}(2)=M+1\right\}} .
$$

\section{Parameters of the Model}

For both coupling schemes, the following inputs are required:

- $M \times(M+1)$ Markovian matrices $P^{(s)}, s=1,2, \ldots, S$;

- a probability distribution $\pi(\cdot)$ on $\{0,1\}^{M S}$;

- an $M \times S$ matrix $Q$ formed by probabilities of success $q_{i, s}$ of Bernoulli random variables in (1).

As it follows from an exhaustive characterization of distributions on binary strings given by Bahadur (1961), relations (3) are not sufficient for identifying a distribution $\pi(\cdot)$ given the transition matrices and vice versa.

Since rating agencies report their Markovian transition matrices, conventionally a matrix $P$, common for all debtors, was assumed to be known and all estimation efforts concentrated on finding $Q$ and $\pi(\cdot)$. Here, following this tradition, matrices $P^{(s)}$ are supposed to be given, while $Q$ and $\pi(\cdot)$ have to be estimated.

Wozabal and Hochreiter (2012) suggested maximum likelihood estimates for these parameters and a heuristic global search method for finding them. Kaniovski and Pflug (2007), for a given $P$, identified a distribution $\pi(\cdot)$, satisfying relations (3), by solving a quadratic programming problem. Conceptually, they searched for a string distribution that is "closest" in the least squares sense to the one with independent tendency variables.

\section{Likelihood Functions and Optimization Problems}

The unknown parameters were estimated by maximizing logarithms of the likelihood functions subject to linear constraints, identical for both coupling schemes.

The likelihood function for the first scheme reads:

$$
\begin{aligned}
L_{1}(\pi(\cdot), Q)= & \prod_{t=1}^{T} \sum_{\vec{\chi} \in\{0,1\}^{M S}} \pi(\vec{\chi}) \prod_{s=1}^{S} \prod_{m_{1}=1}^{M} \prod_{m_{2}=1}^{M+1}\left[q_{m_{1}, s} p_{m_{1}, m_{2}}^{(s)}\right. \\
& \left.+\left(1-q_{m_{1}, s}\right) p_{m_{1}, m_{2}}^{(s)}\left(\chi_{M(s-1)+m_{1}}\right)\right]^{I^{t}\left(s, m_{1}, m_{2}\right)} \\
= & I \prod_{t=1}^{T} \sum_{\vec{\chi} \in\{0,1\}^{M S}} \pi(\vec{\chi}) \prod_{s=1}^{S} \prod_{m_{1}=1}^{M} \prod_{m_{2}=1}^{M+1} f\left(s, \vec{\chi}, m_{1}, m_{2}, Q\right)^{I^{t}\left(s, m_{1}, m_{2}\right)},
\end{aligned}
$$


where

$$
\begin{aligned}
I & =\prod_{t=1}^{T} I(t), \quad I(t)=\prod_{s=1}^{S} \prod_{m_{1}=1}^{M} \prod_{m_{2}=1}^{M+1}\left(p_{m_{1}, m_{2}}^{(s)}\right)^{I^{t}\left(s, m_{1}, m_{2}\right)}, \\
f\left(s, \vec{\chi}, m_{1}, m_{2}, Q\right) & = \begin{cases}\frac{1-q_{m_{1}, s}\left(1-p_{m_{1}}^{(s)}\right)}{p_{m_{1}}^{(s)}} & \text { if } m_{1} \geq m_{2}, \quad \chi_{M(s-1)+m_{1}}=1, \\
\frac{1-q_{m_{1}, s} p_{m_{1}}^{(s)}}{1-p_{m_{1}}^{(s)}} & \text { if } m_{1}<m_{2}, \quad \chi_{M(s-1)+m_{1}}=0, \\
q_{m_{1}, s} & \text { otherwise. }\end{cases}
\end{aligned}
$$

Time instants from $t=1$ through $t=T$ correspond to the period of observation. $I^{t}\left(s, m_{1}, m_{2}\right)$ denotes the number of debtors in industry sector $s$ that have migrated from credit class $m_{1}$ to credit class $m_{2}$ in period $t$.

The likelihood function for the second coupling scheme is as the following:

$$
\begin{aligned}
L_{2}(\pi(\cdot), Q)= & \prod_{t=1}^{T} \sum_{\vec{\chi} \in\{0,1\}^{M S}} \pi(\vec{\chi}) \prod_{s=1}^{S} \prod_{m_{1}=1}^{M} \sum_{m_{2}=1}^{M+1} p_{m_{1}, m_{2}}^{(s)}\left(\chi_{M(s-1)+m_{1}}\right)\left(q_{m_{1}, s} p_{m_{1}, m_{2}}^{(s)}+\right. \\
& \left.+1-q_{m_{1}, s}\right)^{I^{t}\left(s, m_{1}, m_{2}\right)} \prod_{j=1, j \neq m_{2}}^{M+1}\left(q_{m_{1}, s} p_{m_{1}, j}^{(s)}\right)^{I^{t}\left(s, m_{1}, j\right)} \\
= & \prod_{t=1}^{T} I(t) \sum_{\vec{\chi} \in\{0,1\}^{M S}} \pi(\vec{\chi}) \prod_{s=1}^{S} \prod_{m_{1}=1}^{M} g\left(t, s, \vec{\chi}, m_{1}, Q\right) .
\end{aligned}
$$

Here $g\left(t, s, \vec{\chi}, m_{1}, Q\right)$ equals to

$$
\sum_{m_{2}=1}^{M+1} p_{m_{1}, m_{2}}^{(s)}\left(\chi_{M(s-1)+m_{1}}\right)\left(q_{m_{1}, s}+\frac{1-q_{m_{1}, s}}{p_{m_{1}, m_{2}}^{(s)}}\right)^{I^{t}\left(s, m_{1}, m_{2}\right)} \prod_{j=1, j \neq m_{2}}^{M+1} q_{m_{1}, s}^{I^{t}\left(s, m_{1}, j\right)}
$$

There are linear constrains:

$$
\begin{gathered}
\sum_{\substack{\vec{\chi} \in\{0,1\}^{M S} \\
\sum_{\vec{\chi} \in\{0,1\}^{M S}, \chi_{M(s-1)+i}=1} \pi(\vec{\chi})}} \pi(\vec{\chi})=p_{i}^{(s)}, \quad i=1,2, \ldots, M, \quad s=1,2, \ldots, S .
\end{gathered}
$$

Equality (4) states that $\pi(\cdot)$ is a probability distribution. Constraints (5) correspond to relations (3). Conceptually they mean that the coordinate $M(s-1)+i$ of a tendency vector takes on value 1 with probability $p_{i}^{(s)}$. Elements of $Q$ and probabilities $\pi(\cdot)$ belong to $[0,1]$. 
In order to guarantee that all industries move synchronously, the following constraint has to be added:

$$
\sum_{\vec{\chi}=\left(\vec{\chi}^{*}, \vec{\chi}^{*}, \ldots, \vec{\chi}^{*}\right), \vec{\chi}^{*} \in\{0,1\}^{M}} \pi(\vec{\chi})=1 .
$$

It implies that an admissible distribution $\pi(\cdot)$ is nested exclusively on binary vectors consisting of $S$ identical blocks $\vec{\chi}^{*}$ of dimension $M$. Given relations (5), $p_{i}^{(s)}$ cannot depend on $s$ if (6) takes place.

If the Markovian matrices are identical for all industries and the additional constraint (6) holds true, then the first coupling scheme is equivalent to the setting of Wozabal and Hochreiter (2012), while the second coupling scheme corresponds to the setting introduced in Boreiko et al. (2015). The likelihood functions given here can be obtained by a minor modification of the respective arguments in these papers.

If $p_{i, j}^{(s)}=0$ for some $i$ and $j$, then $I^{t}\left(s, m_{1}, m_{2}\right)=0$ as well. Consequently, such terms are excluded from consideration. Also, containing no unknowns, the term $I$ cannot affect a solution of the above maximization problems.

Estimating parameters of a coupled Markov chain model from real data, one deals with a mixture of multinomial distributions. This class of statistical problems is known to imply multiple solutions. A detailed analysis of these problems is given in Allman et al. (2009). However, in practical applications no complications seem to arise. See Carreira-Perpiñán and Renals (2000).

\section{Input Data}

A S\&P's data set covering companies from 30 OECD countries for the period from 1991 through 2013 was considered.

An illustrative example has to deal with a portfolio that is known in everyday practice of risk management. However, the number of industry sectors involved cannot be large for two reasons. First, there must be enough migrations in each industry sector. For example, for estimating the respective Markovian matrix. Second, the dimension of the optimization problem and, consequently, its run time of an optimization algorithm should not explode. Note that $Q$ contains $M \times S$ entries, while a distribution $\pi(\cdot)$ is nested on $2^{M \times S}$ sample points. Hence, the total number of unknowns is $M \times S+2^{M \times S}$.

For example, in September of 2013, in the portfolio generating the Dow Jones iTraxx EUR market index investment grade debtors belonging to five industry sectors were involved: 1 -auto and industrial; 2-consumer; 3-energy with utilities; 4finance and insurance; 5-telecommunications, media and technology. That is, $S=5$. Since there are two non-default credit classes, investment grade and non-investment grade debtors, $M=2$. Then the total number of unknowns is $10+2^{10}=1034$. Investment grade debtors are characterized by S\&P's ratings from $A A A$ to $B B B$, while non-investment grade ones occupy the ratings from $B B$ and downward. Indexes 1,2 and 3 refer to an investment grade, a non-investment grade and a defaulted debtor, respectively. 
With this choice of parameters, using finite-difference approximations of partial derivatives, the corresponding MATLAB software required typically 4-8 hours in order to find a solution. Evaluating the respective derivatives analytically, the runtime reduces drastically. In particular, to a couple of minutes in the case of IP method with first derivatives given in Appendix 2.

Keeping in mind the possibility of multiple solutions, a variety of initial approximations have been tried, including the use of a solution obtained by one of the methods as a starting point for the other one. In all cases the results reported here were identical for both MATLAB algorithms, IP and SQP.

For simulation of defaults, the initial counts were chosen as they were in September of 2013: $N^{1,1}(1)=N^{2,1}(1)=30, N^{3,1}(1)=N^{5,1}(1)=20, N^{4,1}(1)=25$. That is, $\mathcal{N}(1)=125$.

Among all time intervals between 1991 and 2013 that contain sufficiently many credit rating migrations in order to estimate the model parameters, the period from 2000 through 2003 exhibits the greatest probability of default of investment grade debtors for the whole pool of debtors as well as for each of the five industry sectors under consideration. Since the actual iTraxx portfolio contains only debtors of this credit quality, with such choice of a time interval the most intensive flow of defaults should be expected and, thus, it was used for the numerical experiments reported next. Hence, $t=1$ and $T=4$ correspond to years 2000 and 2003, respectively.

The following Markovian transition matrix $P$ is formed by frequencies of all credit rating transitions in the S\&P's data set during the period from 2000 through 2003 , while $P^{(s)}$ contains the respective frequencies characterizing industry sector $s$ :

$$
\begin{aligned}
& P=\left(\begin{array}{lll}
0.9721 & 0.0255 & 0.0024 \\
0.0300 & 0.9157 & 0.0543
\end{array}\right), \quad P^{(1)}=\left(\begin{array}{lll}
0.9417 & 0.0571 & 0.0012 \\
0.0238 & 0.9186 & 0.0577
\end{array}\right), \\
& P^{(2)}=\left(\begin{array}{lll}
0.9546 & 0.0430 & 0.0024 \\
0.0134 & 0.9402 & 0.0464
\end{array}\right), \quad P^{(3)}=\left(\begin{array}{lll}
0.9622 & 0.0336 & 0.0042 \\
0.0492 & 0.9119 & 0.0389
\end{array}\right) \text {, } \\
& P^{(4)}=\left(\begin{array}{lll}
0.9551 & 0.0385 & 0.0064 \\
0.0159 & 0.8924 & 0.0917
\end{array}\right), \quad P^{(5)}=\left(\begin{array}{lll}
0.9805 & 0.0179 & 0.0016 \\
0.0492 & 0.9148 & 0.0360
\end{array}\right) \text {. }
\end{aligned}
$$

\section{Testing Synchronicity}

In the case of synchronously moving industries, the support of a tendency vector consists of the following four sample points:

$$
\text { (0000000000), (0101010101), (1010101010), (1111111111). }
$$

They are formed by the blocks $00,01,10$ and 11 , respectively. Hence, a conclusion concerning synchronicity may be reached by analyzing the support of $\pi(\cdot)$ : every point different from the above four ones is an argument against synchronicity.

In a credit class, correlations between non-deteriorating tendencies governing credit migrations of debtors belonging to different industries can serve as indicators of synchronicity or its lack as well. To this end, consider a $5 \times 5$ matrix $C$ containing below (above) the main diagonal coefficients of correlation 


$$
c_{i, j}=\operatorname{Corr}\left(\Pi_{2(i-1)+1}, \Pi_{2(j-1)+1}\right)\left(\operatorname{Corr}\left(\Pi_{2(i-1)+2}, \Pi_{2(j-1)+2}\right)\right)
$$

between non-deteriorating tendencies affecting investment (non-investment) grade debtors from industry sectors $i$ and $j$. Since

$$
\operatorname{Corr}\left(\Pi_{2(i-1)+1}, \Pi_{2(i-1)+1}\right)=\operatorname{Corr}\left(\Pi_{2(j-1)+2}, \Pi_{2(j-1)+2}\right)=1,
$$

set $c_{i, i}=1$ for all $i$. If industries move synchronously, the above block structure implies that the corresponding coordinates allocated to different industries in a tendency vector coincide. Hence, $c_{i, j}=1$ for all $i \neq j$. Consequently, the number of off-diagonal entries of $C$ that differ from one and the amplitudes of these deviations are numerical measures of synchronicity. The larger they are, the stronger is the evidence that the moves are asynchronous.

As a benchmark case allowing for a comparison with known results, first the parameters were estimated assuming that all industry sectors are governed by the same Markovian matrix.

\subsection{Common for All Industries Transition Matrix $P$}

Let us consider only realizations of the tendency vector whose probabilities exceed the threshold of 0.0001 (0.005). For the first coupling scheme, there are 16 (8) elementary outcomes constituting a sure event (an event occurring with probability 0.9926), while for the second scheme, the corresponding numbers are: 68 (5) and 1.0000 (0.9513). For the first/second coupling scheme matrix $C$ reads:

$\left(\begin{array}{ccccc}1.0000 / 1.0000 & 0.5936 / 0.6219 & 0.6793 / 0.7076 & 0.6630 / 0.6480 & 0.5936 / 0.5348 \\ 0.9999 / 1.0000 & 1.0000 / 1.0000 & 0.8186 / 0.8187 & 0.4565 / 0.4567 & 0.9997 / 0.8348 \\ 0.3144 / 0.3146 & 0.3144 / 0.3146 & 1.0000 / 1.0000 & 0.5476 / 0.5476 & 0.8186 / 0.7148 \\ 0.9999 / 1.0000 & 0.9999 / 1.0000 & 0.3144 / 0.3146 & 1.0000 / 1.0000 & 0.4565 / 0.5395 \\ -0.0287 /-0.0287 & -0.0287 /-0.0287 & 0.3189 / 0.3180 & -0.0287 /-0.0287 & 1.0000 / 1.0000\end{array}\right)$.

The estimated distributions $\pi(\cdot)$ and correlations $c_{i, j}$ exhibit clear asynchronicity patterns. In particular, all $c_{i, j}$ corresponding to non-investment grade debtors and 7 out 10 correlations characterizing investment grade debtors differ from one. (Investment grade debtors from industries 1, 2 and 4 seem to be affected by the same unobserved tendency.) In sum, even if the same transition matrix governs all industry sectors, it appears that the existing coupling schemes with synchronously moving industries do not capture completely the nature of the credit migration process.

A stronger asynchronicity pattern emerged when credit rating migrations in industry sector $s$ were governed by $P^{(s)}$.

\subsection{Industry-Specific Transition Matrices $\boldsymbol{P}^{(s)}$}

Distributions of tendency vectors seem to be identical for both schemes. There are $11(9)$ realizations of the tendency vector whose probabilities exceed $0.0001(0.005)$. 
Table 1 Variation of transition probabilities

\begin{tabular}{lll}
\hline & Upgrading & Downgrading \\
\hline$\chi_{M(s-1)+i}=1$ & $\left(1-q_{i, s}\right) \frac{1-p_{i}^{(s)}}{p_{i}^{(s)}} 100$ & $-\left(1-q_{i, s}\right) 100$ \\
$\chi_{M(s-1)+i}=0$ & $-\left(1-q_{i, s}\right) 100$ & $\left(1-q_{i, s}\right) \frac{p_{i}^{(s)}}{1-p_{i}^{(s)}} 100$ \\
\hline
\end{tabular}

They form a sure event (an event taking place with probability 0.9962). The correlations $c_{i, j}$ are as follows:

$$
\left(\begin{array}{ccccc}
1.0000 & -0.0545 & 0.3831 & 0.7781 & -0.0478 \\
0.6838 & 1.0000 & 0.4326 & 0.1012 & 0.8761 \\
0.4497 & 0.1935 & 1.0000 & 0.2855 & 0.4997 \\
0.4056 & 0.1708 & 0.9141 & 1.0000 & -0.0614 \\
0.5668 & -0.0308 & 0.7115 & 0.6504 & 1.0000
\end{array}\right)
$$

In sum, both the estimated distribution $\pi(\cdot)$ and coefficients of correlation $c_{i, j}$ suggest that the industries in question evolve asynchronously. Since all off-diagonal entries of $C$ differ from 1, it appears that the asynchronicity pattern here is more articulated than in Subsection 6.1, where a common Markovian matrix could act as a factor implying more coherency among hidden tendencies.

The data analyzed here indicate that, allowing for industry specific Markovian matrices and asynchronously evolving industries, more realistic models of dependent credit rating transitions can be developed. However, the documented effect of asynchronously moving industries remains just a "natural phenomenon" as long as no implications regarding riskiness of the portfolio in question have been drawn upon it. A quantitative comparison of the flows of defaults generated by the respective coupling schemes is presented in the next section.

\section{Synchronicity and Riskiness}

Note that a realization of the tendency vector accounts for macroeconomic conditions. If they are favorable, as encoded by "1" at the respective position, migrations towards better/worse credit ratings can be more/less likely than what should be expected according to the corresponding probabilities of the respective Markovian matrix. Adverse conditions, or a " 0 ", incur the opposite effect: less/more likely migrations towards better/worse credit classes. Using relations (1) and (2), the following formulas were obtained for the percentage of these variations in credit class $i$ and industry sector $s$ (Table 1):

Since in our case $p_{i}$ and all $p_{i}^{(s)}$ exceed 0.5 , macroeconomic conditions affect stronger probabilities of a downgrading. For the first coupling scheme, Tables 2 and 3 contain the corresponding percentages in the case of industry-specific and common 
Table 2 Variation of a downgrading probability, industry-specific transition matrices

\begin{tabular}{lrrrrrrrrrr}
\hline $\mathrm{HV} \backslash \mathrm{IS}$ & 1 & \multicolumn{2}{c}{2} & \multicolumn{2}{c}{3} & \multicolumn{3}{c}{5} & \\
\cline { 2 - 10 } & $i=1$ & $i=2$ & $i=1$ & $i=2$ & $i=1$ & $i=2$ & $i=1$ & $i=2$ & $i=1$ & $i=2$ \\
\hline$\chi_{M(s-1)+i}=1$ & 1.53 & 2.75 & 0.79 & 0.83 & 6.3 & 3.4 & 2.38 & 3.39 & 0.97 & 4.07 \\
$\chi_{M(s-1)+i}=0$ & 24.71 & 44.91 & 16.61 & 17.06 & 160.37 & 84.25 & 50.63 & 33.58 & 48.77 & 108.99 \\
\hline \hline
\end{tabular}

Table 3 Variation of a downgrading probability, common for all industries Markovian transition matrix

\begin{tabular}{lrrrrrrrrrr}
\hline $\mathrm{HV} \backslash \mathrm{IS}$ & 1 & \multicolumn{2}{c}{2} & \multicolumn{2}{c}{3} & \multicolumn{2}{c}{4} & \multicolumn{2}{l}{5} \\
\cline { 2 - 11 } & $i=1$ & $i=2$ & $i=1$ & $i=2$ & $i=1$ & $i=2$ & $i=1$ & $i=2$ & $i=1$ & $i=2$ \\
\hline$\chi_{M(s-1)+i}=1$ & 3.87 & 2.87 & 1.74 & 14.54 & 6.98 & 28.30 & 2.66 & 5.45 & 30.07 & 33.62 \\
$\chi_{M(s-1)+i}=0$ & 62.51 & 47.04 & 28.11 & 238.67 & 112.75 & 463.59 & 42.97 & 89.28 & 485.71 & 550.07 \\
\hline \hline
\end{tabular}

for all debtors Markovian matrices, respectively. The values $q_{i, s}$ used for calculations are given in Appendix 1.

A downgrading for an investment grade debtor implies either a migration to the non-investment grade category or a default, while for a non-investment grade debtor it means a default. Abbreviations HV and IS stand for hidden variable and industry sector.

Note that there are models of credit rating migrations, where a Markovian matrix, common for all industries, is adjusted according to macroeconomic conditions. See among others Bangia et al. (2002), McNeil and Wendin (2007), Frydman and Schuermann (2008), Korolkiewicz and Elliott (2008), Stefanescu et al. (2009) and Xing et al. (2012).

Since all but two values in Table 3 exceed their counterparts of Table 2, the situation when a unique Markovian matrix governs migrations in all industry sectors should generate a stronger flow of defaults as compared with the case of industry-specific transition matrices. This guess is confirmed next by simulating defaults according to the above coupling schemes and analyzing the corresponding sample distributions.

Let us consider time periods of 1,5 and 7 years. Each of the sample values given below was obtained with 100,000 independent observations. The parameters used for simulations are summarized in Appendix 1. Abbreviations AS, AC and SC stand for asynchronously moving industries governed by industry-specific Markovian matrices, asynchronously moving industries governed by a common transition matrix, and synchronously moving industries governed by a common Markovian matrix, respectively. The SC case corresponds to the known coupling schemes. It serves as a benchmark here (Tables 4, 5, 6).

In the case of asynchronously moving industries, greater $C V a R$ values (eleven out of twelve) for all three time horizons suggest, that the loss distribution has a heavier tail when migrations in all industries are governed by the same $P$. The respective percentiles exhibit the same pattern. 
Table 4 VaR and CVaR of loss, 1 year

\begin{tabular}{lllll}
\hline Scheme $\backslash$ percentile & 50 & 75 & 90 and $C \operatorname{VaR}(0.1)$ & 95 and $C \operatorname{VaR}(0.05)$ \\
\hline AS & $0.59 / 0.59$ & $1.01 / 1.01$ & 1.47 and $1.71 / 1.47$ and 1.71 & 1.69 and $2.49 / 1.71$ and 2.47 \\
AC & $0.51 / 0.50$ & $0.77 / 0.75$ & 1.35 and $2.13 / 1.26$ and 3.38 & 1.58 and $2.13 / 1.49$ and 3.38 \\
SC & $0.51 / 0.50$ & $0.76 / 0.75$ & 1.33 and $2.42 / 1.27$ and 3.67 & 1.56 and $2.42 / 1.50$ and 3.67 \\
\hline
\end{tabular}

Table 5 VaR and CVaR of loss, 5 years

\begin{tabular}{lllll}
\hline Scheme $\backslash$ percentile & 0.5 & 0.75 & 0.9 and $C \operatorname{VaR}(0.1)$ & 0.95 and $C \operatorname{VaR}(0.05)$ \\
\hline AS & $3.69 / 3.69$ & $4.84 / 4.83$ & 6.02 and $6.96 / 6.02$ and 6.94 & 6.74 and $7.68 / 6.73$ and 7.67 \\
AC & $2.14 / 2.13$ & $3.46 / 3.15$ & 5.46 and $7.88 / 4.68$ and 7.77 & 7.08 and $9.77 / 6.73$ and 10.43 \\
SC & $2.00 / 2.12$ & $3.17 / 3.17$ & 5.88 and $9.73 / 4.98$ and 9.67 & 8.17 and $12.84 / 8.21$ and 12.31 \\
\hline
\end{tabular}

Table 6 VaR and CVaR of loss, 7 years

\begin{tabular}{lllll}
\hline Scheme $\backslash$ percentile & 0.5 & 0.75 & 0.9 and $C \operatorname{VaR}(0.1)$ & 0.95 and $C \operatorname{VaR}(0.05)$ \\
\hline AS & $6.03 / 6.03$ & $7.52 / 7.52$ & 8.87 and $10.31 / 8.93$ and 11.31 & 9.77 and $11.06 / 9.82$ and 11.06 \\
AC & $3.45 / 3.42$ & $5.39 / 4.85$ & 8.16 and $11.90 / 7.30$ and 11.28 & 10.39 and $13.58 / 10.11$ and 13.32 \\
SC & $3.16 / 3.44$ & $5.09 / 5.00$ & 9.16 and $14.03 / 8.61$ and 12.88 & 11.76 and $17.43 / 12.18$ and 16.13 \\
\hline
\end{tabular}

Observe that, the larger an entry of $Q$ is, the weaker will be, on the one hand, the dependence of the respective asset from the rest of the portfolio and, on the other hand, the effect of macroeconomic conditions on the respective transition probabilities. Given in Appendix 1 entries corresponding to industry-specific $P^{(s)}$ exceed their counterparts for a single $P$. Consequently, as compared with the case of a common $P$, first, the dependence among assets is weaker and, second, the downgrading probabilities increase less. Both factors together contribute toward higher losses the case of a single $P$.

Comparing patterns corresponding to asynchronously and synchronously moving industries governed by the same transition matrix, a heavier tail of the loss distribution for all three time horizons can be attributed to the cascade effect discussed in Kaniovski and Pflug (2007) for the setting with synchronously moving industries.

\section{Conclusions}

Simulations based on S\&P's data show lack of synchronicity among hidden variables shaping credit rating migrations in different industry sectors. This phenomenon indicates, in particular, that macroeconomic conditions affect differently the five industry sectors considered in the paper. It would be interesting to compare for different rating 
agencies patterns of dependence between hidden variables and, consequently, strength and direction of the corresponding macroeconomic factors.

Open Access This article is distributed under the terms of the Creative Commons Attribution 4.0 International License (http://creativecommons.org/licenses/by/4.0/), which permits unrestricted use, distribution, and reproduction in any medium, provided you give appropriate credit to the original author(s) and the source, provide a link to the Creative Commons license, and indicate if changes were made.

\section{Appendix 1}

In each case of asynchronously moving industries, simulations were run for the distribution $\pi(\cdot)$ corresponding to the threshold of 0.005 . They are presented here. Since the sum of probabilities of the respective elementary outcomes is less than 1 , the values quoted were normalized.

Industry-specific matrices $P^{(s)}$. The following $Q$ was obtained for the first/second coupling scheme:

$$
\left(\begin{array}{lllll}
0.9847 / 0.9909 & 0.9921 / 0.9963 & 0.9370 / 0.9495 & 0.9762 / 0.9816 & 0.9903 / 0.9909 \\
0.9725 / 0.9729 & 0.9917 / 0.9925 & 0.9659 / 0.9645 & 0.9661 / 0.9665 & 0.9593 / 0.9660
\end{array}\right) .
$$

Distribution $\pi(\cdot)$ is the same in both cases:

$$
\begin{aligned}
& \pi(0011000001)=0.0196, \pi(0100101110)=0.0195, \pi(0100111110)=0.0167 \\
& \pi(1011111011)=0.0382, \pi(1100010011)=0.0094, \pi(1111010011)=0.0051 \\
& \pi(1111110011)=0.0071, \pi(1111111011)=0.0087, \pi(1111111111)=0.8756 .
\end{aligned}
$$

Asynchronously moving industries, common transition matrix P. Distribution $\pi(\cdot)$ for the first scheme reads:

$$
\begin{aligned}
& \pi(0001010011)=0.0094, \pi(0001110011)=0.0116, \pi(0101110011)=0.0071, \\
& \pi(1010001100)=0.0160, \pi(1010101010)=0.0072, \pi(1010101100)=0.0082, \\
& \pi(1110101010)=0.0159, \pi(1111111111)=0.9246,
\end{aligned}
$$

while for the second scheme it is:

$$
\begin{aligned}
& \pi(0001000011)=0.0098, \pi(0001110011)=0.0106, \pi(0101110010)=0.0089, \\
& \pi(1010101100)=0.0059, \pi(1111111111)=0.9648 .
\end{aligned}
$$

For the first/second coupling scheme the following $Q$ was obtained:

$$
\left(\begin{array}{lllll}
0.9613 / 0.9643 & 0.9826 / 0.9855 & 0.9302 / 0.9438 & 0.9734 / 0.9789 & 0.6993 / 0.6993 \\
0.9713 / 0.9681 & 0.8545 / 0.7097 & 0.7170 / 0.9505 & 0.9455 / 0.9451 & 0.6638 / 0.9643
\end{array}\right) .
$$


As a benchmark, in the case of synchronously moving industries, $Q$ for the first/second coupling scheme is:

$$
\left(\begin{array}{lllll}
0.9600 / 0.9662 & 0.9890 / 0.9865 & 0.9302 / 0.9438 & 0.9619 / 0.9780 & 0.6990 / 0.6993 \\
0.9706 / 0.9674 & 0.8496 / 0.7101 & 0.9622 / 0.9504 & 0.9378 / 0.9356 & 0.6646 / 0.9587
\end{array}\right)
$$

and the same distribution $\pi(\cdot)$ was obtained in both cases:

$$
\begin{aligned}
& \pi(0000000000)=0.0279, \pi(1010101010)=0.0264 \\
& \pi(1111111111)=0.9457 .
\end{aligned}
$$

\section{Appendix 2}

Consider first coupling scheme 1. Set

$$
F_{1}(t)=\sum_{\vec{\chi} \in\{0,1\}^{M S}} \pi(\vec{\chi}) G_{1}(t, \vec{\chi})
$$

where

$$
G_{1}(t, \vec{\chi})=\prod_{s=1}^{S} \prod_{m_{1}=1}^{M} \prod_{m_{2}=1}^{M+1} f\left(s, \vec{\chi}, m_{1}, m_{2}, Q\right)^{I^{t}\left(s, m_{1}, m_{2}\right)}
$$

Since

$$
\ln L_{1}(\pi(\cdot), Q)=\sum_{t=1}^{T} \ln F_{1}(t)+\sum_{t=1}^{T} \ln I(t)
$$

and the second sum here does not contain the unknowns, it is enough to evaluate derivatives of $\ln F_{1}(t)$.

To this end

$$
\begin{aligned}
\frac{\partial \ln F_{1}(t)}{\partial \pi(\vec{\chi})} & =\frac{1}{F_{1}(t)} G_{1}(t, \vec{\chi}) \quad \text { and } \quad \frac{\partial \ln F_{1}(t)}{\partial q_{m, i}} \\
& =\frac{1}{F_{1}(t)} \sum_{\vec{\chi} \in\{0,1\}^{M S}} \pi(\vec{\chi}) \frac{\partial G_{1}(t, \vec{\chi})}{\partial q_{m, i}}
\end{aligned}
$$


By using the logarithmic differentiation and taking into account that, whenever $s \neq i$ or $m_{1} \neq m$, the partial derivative of the corresponding terms is zero, it follows that

$$
\begin{aligned}
\frac{\partial G_{1}(t, \vec{\chi})}{\partial q_{m, i}} & =G_{1}(t, \vec{\chi}) \sum_{s=1}^{S} \sum_{m_{1}=1}^{M} \sum_{m_{2}=1}^{M+1} \frac{\partial}{\partial q_{m, i}} \ln \left[f\left(s, \vec{\chi}, m_{1}, m_{2}, Q\right)^{I^{t}\left(s, m_{1}, m_{2}\right)}\right] \\
& =G_{1}(t, \vec{\chi}) \sum_{m_{2}=1}^{M+1} \frac{\partial}{\partial q_{m, i}} \ln \left[f\left(i, \vec{\chi}, m, m_{2}, Q\right)^{I^{t}\left(i, m, m_{2}\right)}\right] \\
& =G_{1}(t, \vec{\chi}) \sum_{m_{2}=1}^{M+1} I^{t}\left(i, m, m_{2}\right) \frac{1}{f\left(i, \vec{\chi}, m, m_{2}, Q\right)} \frac{\partial}{\partial q_{m, i}} f\left(i, \vec{\chi}, m, m_{2}, Q\right) .
\end{aligned}
$$

Here

$$
\frac{\partial}{\partial q_{m, i}} f\left(i, \vec{\chi}, m, m_{2}, Q\right)= \begin{cases}\frac{p_{m}^{(i)}-1}{p_{m}^{(i)}} & \text { if } m \geq m_{2}, \chi_{M(i-1)+m}=1, \\ \frac{p_{m}^{(i)}}{p_{m}^{(i)}-1} & \text { if } m<m_{2}, \chi_{M(i-1)+m}=0 \\ 1 & \text { otherwise. }\end{cases}
$$

In the same way, for scheme 2, it is sufficient to evaluate derivatives of

$$
F_{2}(t)=\sum_{\vec{\chi} \in\{0,1\}^{M S}} \pi(\vec{\chi}) G_{2}(t, \vec{\chi}),
$$

where

$$
G_{2}(t, \vec{\chi})=\prod_{s=1}^{S} \prod_{m_{1}=1}^{M} \sum_{m_{2}=1}^{M+1} g\left(t, s, \vec{\chi}, m_{1}, Q\right) .
$$

Analogously to the previous case

$$
\begin{aligned}
\frac{\partial \ln F_{2}(t)}{\partial \pi(\vec{\chi})} & =\frac{1}{F_{2}(t)} G_{2}(t, \vec{\chi}) \quad \text { and } \quad \frac{\partial \ln F_{2}(t)}{\partial q_{m, i}} \\
& =\frac{1}{F_{2}(t)} \sum_{\vec{\chi} \in\{0,1\}^{M S}} \pi(\vec{\chi}) \frac{\partial G_{2}(t, \vec{\chi})}{\partial q_{m, i}}
\end{aligned}
$$

where

$$
\begin{aligned}
\frac{\partial G_{2}(t, \vec{\chi})}{\partial q_{m, i}} & =G_{2}(t, \vec{\chi}) \sum_{s=1}^{S} \sum_{m_{1}=1}^{M} \frac{\partial}{\partial q_{m, i}} \ln g\left(t, s, \vec{\chi}, m_{1}, Q\right) \\
& =G_{2}(t, \vec{\chi}) \frac{\partial}{\partial q_{m, i}} \ln g(t, i, \vec{\chi}, m, Q) \\
& =G_{2}(t, \vec{\chi}) \frac{1}{g(t, i, \vec{\chi}, m, Q)} \frac{\partial g(t, i, \vec{\chi}, m, Q)}{\partial q_{m, i}},
\end{aligned}
$$




$$
\begin{aligned}
& \frac{\partial g(t, i, \vec{\chi}, m, Q)}{\partial q_{m, i}}=\sum_{m_{2}=1}^{M+1} p_{m_{1}, m_{2}}^{(s)}\left(\chi_{M(s-1)+m_{1}}\right) \\
& \times \frac{\partial}{\partial q_{m, i}}\left(q_{m, i}+\frac{1-q_{m, i}}{p_{m, m_{2}}^{(i)}}\right)^{I^{t}\left(i, m, m_{2}\right)} \prod_{j=1, j \neq m_{2}}^{M+1} q_{m, i}^{I^{t}(i, m, j)}, \\
& \frac{\partial}{\partial q_{m, i}}\left[\left(q_{m, i}+\frac{1-q_{m, i}}{p_{m, m_{2}}^{(i)}}\right)^{I^{t}\left(i, m, m_{2}\right)} \prod_{j=1, j \neq m_{2}}^{M+1} q_{m, i}^{I^{t}(i, m, j)}\right] \\
& =\left[\left(q_{m, i}+\frac{1-q_{m, i}}{p_{m, m_{2}}^{(i)}}\right)^{I^{t}\left(i, m, m_{2}\right)} \prod_{j=1, j \neq m_{2}}^{M+1} q_{m, i}^{I^{t}(i, m, j)}\right] \\
& \times \frac{\partial}{\partial q_{m, i}} \ln \left[\left(q_{m, i}+\frac{1-q_{m, i}}{p_{m, m_{2}}^{(i)}}\right)^{I^{t}\left(i, m, m_{2}\right)} \prod_{j=1, j \neq m_{2}}^{M+1} q_{m, i}^{I^{t}(i, m, j)}\right], \\
& \frac{\partial}{\partial q_{m, i}} \ln \left[\left(q_{m, i}+\frac{1-q_{m, i}}{p_{m, m_{2}}^{(i)}}\right)^{I^{t}\left(i, m, m_{2}\right)} \prod_{j=1, j \neq m_{2}}^{M+1} q_{m, i}^{I^{t}(i, m, j)}\right] \\
& =I^{t}\left(i, m, m_{2}\right) \frac{\partial}{\partial q_{m, i}} \ln \left(q_{m, i}+\frac{1-q_{m, i}}{p_{m, m_{2}}^{(i)}}\right) \\
& +\left[\sum_{j=1, j \neq m_{2}}^{M+1} I^{t}(i, m, j)\right] \frac{\partial \ln q_{m, i}}{\partial q_{m, i}} \\
& =I^{t}\left(i, m, m_{2}\right) \frac{1-\frac{1}{p_{m, m_{2}}^{(i)}}}{q_{m, i}+\frac{1-q_{m, i}}{p_{m, m_{2}}^{(i)}}}+\left[\sum_{j=1, j \neq m_{2}}^{M+1} I^{t}(i, m, j)\right] \frac{1}{q_{m, i}} .
\end{aligned}
$$

\section{References}

Allman, E. L., Matias, C., \& Rhodes, J. A. (2009). Identifiability of parameters in latent structure with many observed variables. Annals of Statistics, 37(6A), 3099-3132.

Bahadur, R. R. (1961). A representation of the joint distribution of responses to $n$ dichotomous items. In H. Solomon (Ed.), Studies in item analysis and prediction, Stanford University Press, USA, 158-168.

Bangia, A., Diebold, F. X., Kronimus, A., Schagen, C., \& Schuermann, T. (2002). Ratings migration and the business cycle, with applications to credit portfolio stress testing. Journal of Banking and Finance, 26(2/3), 445-474.

Boreiko, D. V., Kaniovski, Y. M., \& Pflug, G. Ch. (2015). Modeling dependent credit rating transitions-A comparison of coupling schemes and empirical evidence. Central European Journal of Operations Research,. doi:10.1007/s10100-015-0415-6.

Carreira-Perpiñán, M., \& Renals, S. (2000). Practical identifiability of finite mixtures of multivariate Bernoulli distributions. Neural Computation, 12(1), 141-152.

Choroś-Tomczyk, B., Härdle, W., \& Okhrin, O. (2013). Valuation of collateralized debt obligations with hierarchical Archimedean copulae. Journal of Empirical Finance, 24(1), 42-62.

Frydman, H., \& Schuermann, T. (2008). Credit rating dynamics and Markov mixture models. Journal of Banking and Finance, 32(6), 1062-1075. 
Gupton, G. M., Finger, Ch C, \& Bhatia, M. (1997). CreditMetrics-Technical document. New York: J.P. Morgan Inc.

Hull, J., \& White, A. (2004). Valuation of a CDO and an $n$-th to default CDS without Monte Carlo simulation. Journal of Derivatives, 12(2), 8-23.

Kaniovski, Y. M., \& Pflug, G Ch. (2007). Risk assessment for credit portfolios: A coupled Markov chain model. Journal of Banking and Finance, 31(8), 2303-2323.

Korolkiewicz, M., \& Elliott, R. (2008). A hidden Markov model of credit quality. Journal of Economic Dynamics and Control, 32(12), 3807-3819.

Li, D. X. (2000). On default correlation: A copula approach. Journal of Fixed Income, 9(4), 43-54.

McNeil, A., \& Wendin, J. (2007). Bayesian inference for generalized linear mixed models of portfolio credit risk. Journal of Empirical Finance, 14(2), 131-149.

Nagpal, K., \& Bahar, R. (2001). Measuring default correlation. Risk, 14(3), 129-132.

Stefanescu, C., Tunary, R., \& Turnbull, S. (2009). The credit rating process and estimation of transition probabilities: A Bayesian approach. Journal of Empirical Finance, 16(2), 216-234.

Wozabal, D., \& Hochreiter, R. (2012). A coupled Markov chain approach to credit risk modeling. Journal of Economic Dynamics and Control, 36(3), 403-415.

Xing, H., Sun, N., \& Chen, Y. (2012). Credit rating dynamics in the presence of unknown structural breaks. Journal of Banking and Finance, 36(1), 78-89. 